\title{
Current status of the erector spinae plane block
}

\author{
Yasuhiro Morimoto, MD, \\ Department of Anesthesia, Ube Industries Central Hospital, Ube Yamaguchi, Japan
}

Correspondence: Yasuhiro Morimoto, MD, 750-Nishikiwa, Ube Yamaguchi, Japan 755-0151; E-Mail: yasumorimo@gmail.com

\section{Abstract}

Erector spinae plane block (ESPB) was first reported as a technique, blocking the posterior ramus of the spinal nerves to obtain analgesia in the back. After the original report, many reports have suggested that ESPB could obtain a broader analgesic effect than initially thought and be effective in thoracic or abdominal surgery. By the ESPB, the local anesthetic spreads laterally and blocks the lateral cutaneous branch of the intercostal nerve. Another possibility is the local anesthetic infiltrates ventrally and migrates to the paravertebral space or epidural space. However, the results of the cadaver examination, whether the local anesthetics can reach the paravertebral space were inconsistent. Recently, the clinical effectiveness of ESPB has been reported in thoracic surgery and abdominal surgery. However, the clinical effect of ESPB might be only due to an increase in the blood concentration of local anesthetics. The ideal target might be a deeper site than that currently used in ESPB to reach the paravertebral space. Further studies will investigate more effective and reliable sites for pain relief by injecting local anesthetics around the erector spinae muscle.

Key words: Erector spinae plane block; Local anesthetics; Nerve block

Citation: Morimoto Y. Current status of the erector spinae plane block. Anaesth. Pain intensive care 2021;25(1):83-87. DOI: 10.35975/apic.v25i1.1435

Received: 15 January 2021, Reviewed: 16 January 2021, Accepted: 16 January 2021

\section{Introduction}

The progress of ultrasound-guided peripheral nerve blocks has been remarkable. Recently, inter-fascial plane block has been the focus of attention. Among the novel inter-fascial plane block techniques, the erector spinae plane block (ESPB) has attracted particular attention. ESPB might be a remarkable block in which a local anesthetic administered to the lateral portion of the transverse process of the thoracic vertebrae is effective not only in the back and lateral thorax but also in the ventral median region. In this manuscript, I summarize the findings to date, whether such an effect can be achieved with ESPB.

\section{First report of ESPB}

ESPB was first reported by Forero et al. as a technique, blocking the posterior ramus of the spinal nerves to obtain analgesia in the back. ${ }^{1}$ In the original method, two administration sites were described: deep in the erector spinae muscle or lateral to the transverse process of the spine or superficial of the erector spinae muscle. However, the method targeting deep in the erector spinae muscle became the standard. After the original report, many reports have suggested that ESPB could obtain a broader analgesic effect than initially thought and be effective in thoracic or abdominal surgery. ${ }^{2,3}$

\section{Mechanism of analgesia}

Theoretically, ESPB is effective in blocking the posterior ramus of spinal nerves. However, the mechanism of ESPB in other areas is elusive.

One possibility is that the local anesthetic spreads laterally and blocks the lateral cutaneous branch of the intercostal nerve (mechanism 1$).{ }^{4}$ The other possibility is that the local anesthetic infiltrates ventrally and migrates to the paravertebral space or epidural space (mechanism 2). ${ }^{5}$

\subsection{Mechanism 1}

The site of local anesthetic administration of ESPB is 
the deeper part of the erector spinae muscle. If this plane is traced laterally, it reaches the area between the external intercostal muscle and serratus anterior muscle. This plane is also the site of local anesthetic administration for the serratus plane block or pectoral nerve (PECS) block (Figure 1, 2). ${ }^{4}$

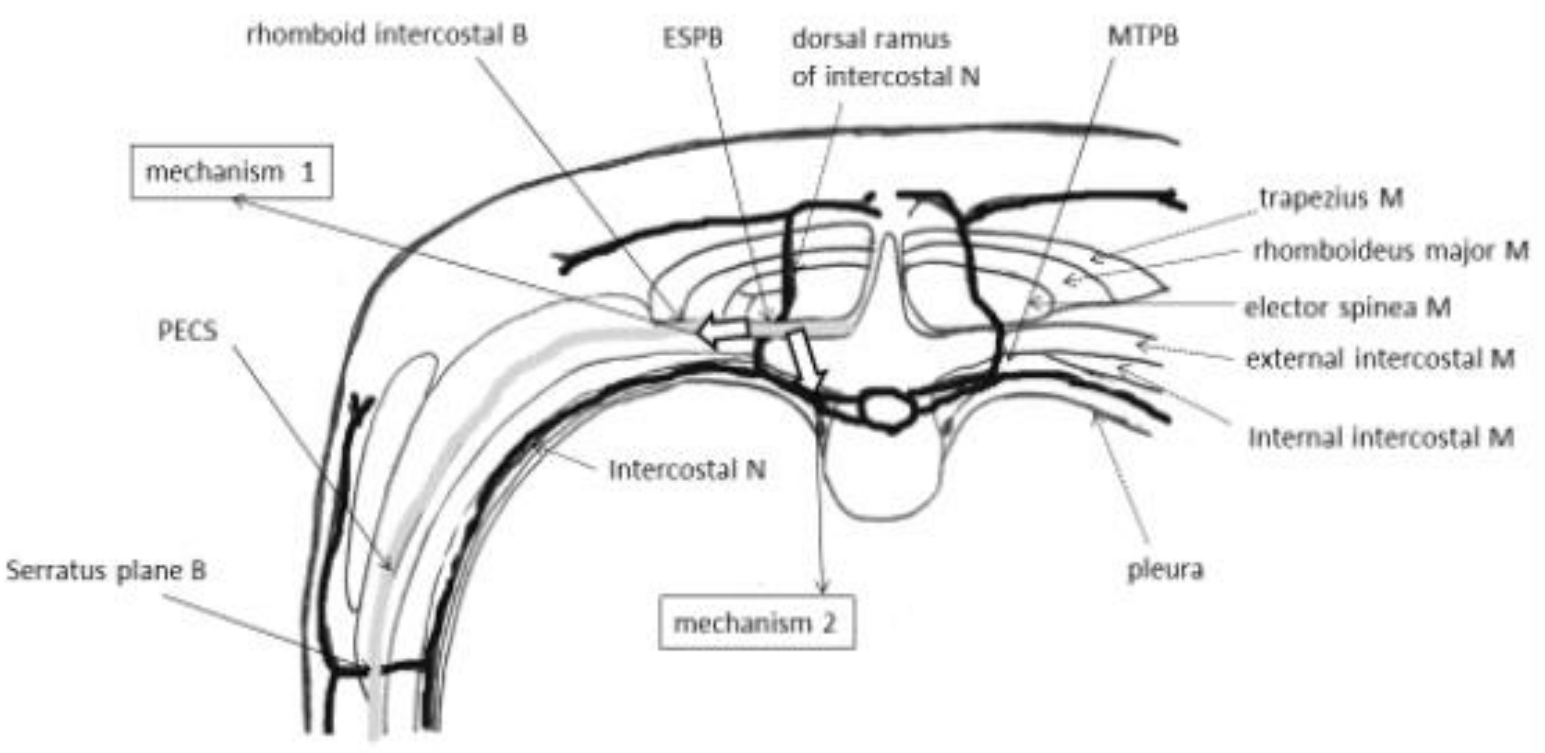

\section{Figure 1: Anatomy around the elector spinae muscle}

The local anesthetic injected by ESPB might spread laterally and blocks the lateral cutaneous branch of the intercostal nerve (mechanism 1) or local anesthetic infiltrates ventrally and migrates to the paravertebral space or epidural space (mechanism 2). M: muscle; N: Nerve; B: Block; ESPB: elector spinae plane block; MTPB: midpoint transverse process to the pleura block

Taketa et al. reported that the analgesic range of the ESPB is the lateral aspect of the thorax, excluding the peri-sternal area. ${ }^{6}$ This result suggests that the mechanism 1 is correct. However, analgesia in the anterior cutaneous branch of the intercostal nerve is necessary for surgery through the abdominal midline incision. It cannot be explained by this mechanism.

\subsection{Mechanism 2}

The second mechanism is that the local anesthetic infiltrates ventrally to reach the paravertebral or epidural space. In other words, ESPB might be compatible with the paravertebral block (PVB).

If the local anesthetic can reach the paravertebral cavity with ESPB, it would be sufficient for a median incision in an abdominal surgery. PVB is an effective block for analgesia of the chest and abdomen.

However, the technique is somewhat difficult, and there is a risk of pneumothorax. ESPB is easier to perform than PVB because the target is more superficial, and the longer distance to the pleura reduces the risk of pneumothorax. Therefore, ESPB has become a promising alternative to $\mathrm{PVB}^{7}$

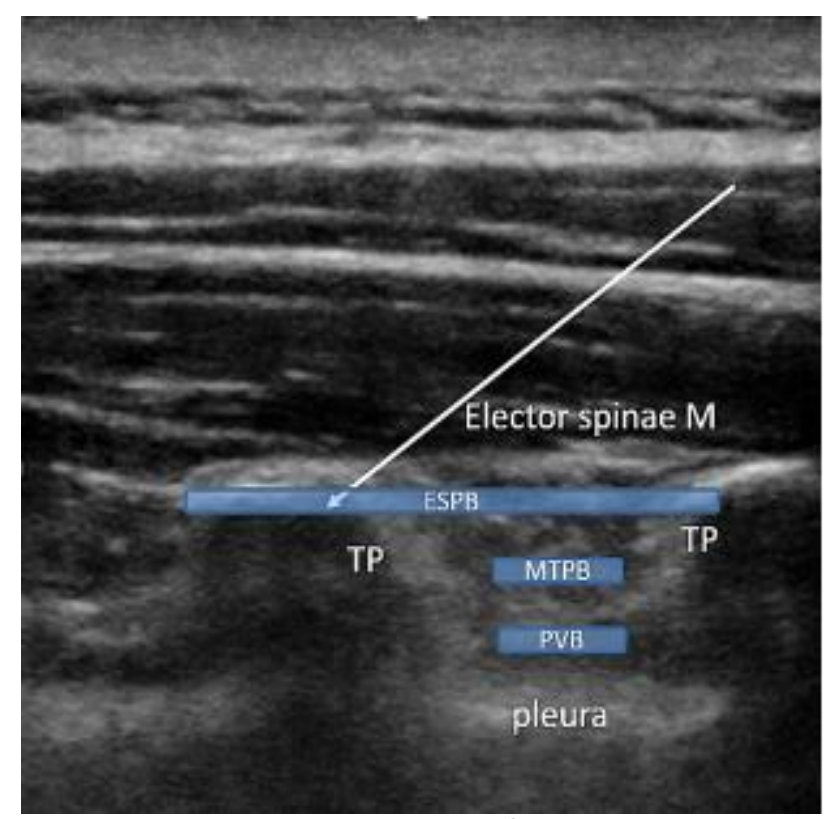

Figure 2: Ultrasound image for ESPB, MTPB or PVB

MTPB: the midpoint transverse process to the pleura block, PVB: paravertebral block, TP: the transverse process 


\section{Results of the cadaver studies}

Several studies have examined the spread of ESPB in cadavers. Adhikary et al. performed a unilateral ESPB and retrolaminar block at the level of T5 with $20 \mathrm{~mL}$ of a dye. ${ }^{8}$ Retrolaminar block is a similar approach with ESPB that blocks the dorsal ramus of spinal nerves. When the spread of the dye was examined by magnetic resonance imaging or dissection, both ESPB and retrolaminar block had spread into the epidural space. ESPB also spread to the lateral intercostal space, suggesting direct blocking of the intercostal nerve. However, this study has been criticized because both blocks were performed at the same level. Furthermore, it was not possible to distinguish which effect was responsible for the spread to the epidural space.

\section{Clinical effectiveness in thoracic surgery}

Fang et al. compared the postoperative status of openchest surgery between ESPB and thoracic paravertebral block (TPVB) $(0.25 \%$ bupivacaine 20 $\mathrm{mL}$ at $\mathrm{T} 5$ level). ${ }^{10}$ Postoperatively, sufentanil intravenous patient-controlled analgesia (IV-PCA) was used. The results showed no difference in the visual analog scale at rest and when coughing, between the groups. Hypotension and bradycardia were more common in TPVB. Moreover, block procedure time and single puncture success rate were better in ESPB. This study concluded that ESPB was easier to perform than TPVB, provided similar analgesia and had fewer complications. However, since IV-PCA was used postoperatively, the effect of systemic administration of opioids on the effectiveness

\section{Table 1: Results of cadaver studies on ESPB}

\begin{tabular}{|l|l|c|c|}
\hline \multicolumn{1}{|c|}{ Author } & \multicolumn{1}{c|}{ Journal } & Block site & $\begin{array}{c}\text { Spread to the } \\
\text { paravertebral } \\
\text { space }\end{array}$ \\
\hline Aponte A, et al. & Can J Aaesth 2019;66:886 & T7 & - \\
\hline Elsharkawy H, et al. & Reg Anesth Pain Med 2019; & T10-11 & - \\
\hline Ivanusic J, et al. & Reg Anesth Pain Med 2018;43:567 & T5 & - \\
\hline Altinpulluk EY, et al. & Rom J Anaesth Intensive Care 2019;26:83 & T9 & + \\
\hline Vidal E, et al. & Rev Esp Anesthesiol Reanim 2018;65:514 & T4, T5, T10 & + \\
\hline Yang H, et al. & Anesthesia 2018;73:1244 & T5 & + \\
\hline
\end{tabular}

Alternatively, Ivanusic et al. studied the spread of ESPB at the level of T5 in a cadaver before fixation. ${ }^{9}$ Twenty milliliters of dye injection showed lateral spread in the superficial and deep layers of the erector spinae muscle. The posterior ramus of the spinal nerves were stained up to the dorsal side of the costotransverse ligament. However, the intercostal nerve was not stained ventrally, and spread into the paravertebral space was not confirmed.

Thus, the results of the cadaver examination were inconsistent (Table 1). This difference may be due to differences in cadavers and subtle differences in the needle tip positions. Additionally, factors such as muscle tension, blood flow, and intrathoracic pressure fluctuations differ between patients and cadavers, limiting the ability to elucidate the mechanism of action of ESPB in these studies. of the block should be considered.

\section{Volunteer studies}

Recently, there have been some negative opinions about the effects of ESPB based on the results of volunteer studies.

Zhang et al. examined the effects of ESPB in 12 volunteers. After administering $20 \mathrm{~mL}$ of $0.5 \%$ ropivacaine at the $\mathrm{T} 5$ level, the area of anesthesia on the blocked side was limited to the back to the posterior axillary line, and there was no effect on the lateral or anterior thoracic wall. ${ }^{11}$ Byrne et al. similarly examined six volunteers. After administering $30 \mathrm{~mL}$ of $0.375 \%$ ropivacaine, a decrease in the perception in the posterior wall only was observed in three volunteers. ${ }^{12}$ However, in three volunteers, the effect 
was observed from the lateral to the anterior wall.

Thus, the effects of ESPB vary from person to person and may also depend on the skill of the practitioner and amount of local anesthetic administered. Based on these considerations, Lonnqvist et al. published a contrasting report that the clinical effect of ESPB is only due to an increase in the blood concentration of local anesthetics. ${ }^{13}$ Systemic administration of local anesthetics, such as lidocaine, is as effective as multimodal analgesia. ESPB uses a large amount of local anesthetic; the possibility cannot be denied that locally administered local anesthetics are absorbed and produce analgesic effects in synergy with systemically administered opioids.

\section{Modifications of ESPB}

Several improvements have been reported to ensure the effectiveness of ESPB to reach the paravertebral space by mechanism 2 .

The midpoint transverse process to the pleura (MTP) block targets the midpoint between the transverse process and the pleura. ${ }^{14}$ Moreover, it is a method for administering local anesthetic slightly deeper than ESPB. MTP block may be more effective if it emphasizes the extension into the paraspinal space, but few reports still exist. Similarly, Shibata et al. presented the costotransverse foramen block as an intermediate technique between ESPB and TPVB. ${ }^{15}$ They administered local anesthetic by advancing the needle below the base of the transverse process of the thoracic spine. In a cadaver study, they confirmed the spread of local anesthetics to the thoracic paravertebral space. These reports suggest that the ideal target might be a deeper site than that which is currently used in ESPB to reach the paravertebral space.

As presented in this manuscript, the usefulness of ESPB is conflicting. ESPB is certainly effective in back surgeries, including spinal surgery. To achieve a wider effect of analgesia, the technique might need to be modified. The ideal target to expect mechanism 2 is slightly deeper than the erector spinae muscles, including the MTP block or costotransverse foramen block. These approaches should be effective for abdominal surgery or midline incision in cardiac surgery.

If mechanism 1 is used, a more lateral approach including a rhomboid intercostal block should be considered. ${ }^{16}$ These approaches should be effective for thoracic surgery, including video-assisted thoracic surgery. The approach should be modified based on ESPB, depending on the surgery.

\section{Conclusion}

In conclusion, ESPB is not a miracle method to obtain widespread analgesia with a simple technique. However, it is strongly believed that ESPB will have clinical significance. Further studies will investigate more effective and reliable sites for pain relief by injecting local anesthetics around the erector spinae muscle.

\section{Conflict of interest}

The author has no conflict of interest to declare

\section{References}

1. Forero M, Adhikary SD, Lopez H, Tsui C, Chin KJ. The erector spinae plane block: a novel analgesic technique in thoracic neuropathic pain. Reg Anesth Pain Med 2016;41:621-627.

[PubMed] DOI: 10.1097/AAP.0000000000000451

2. Forero $\mathrm{M}$, Rajarathinam M, Adhikary $\mathrm{S}$, Chin KJ. Continuous erector plane block for rescue analgesia in thoracotomy after epidural failure : a case report. A A Case Rep 2017:8:54-56. [PubMed] DOI: 10.1213/XAA.0000000000000478

3. Chin KJ, Malhas L, Perlas A. The erector spinae plane block provides visceral abdominal analgesia in bariatric surgery: a report of 3 cases. Reg Anesth Pain Med 2017;42:372-376.

[PubMed] DOI: $10.1097 / A A P .0000000000000581$

4. Elsharkawy H, Pawa A, Mariano ER. Interfascial plane blocks: back to basics. Reg Anesth Pain Med 2018;43:341-6.

[PubMed] DOI: 10.1097/AAP.0000000000000750

5. Onishi E, Toda N, Kameyama Y, Yamauchi M. Comparison of clinical efficacy and anatomical investigation between retrolaminar block and erector spinae plane block. Biomed Res Int 2019:; 2019:2578396. [PubMed] DOI: 10.1155/2019/2578396

6. Taketa $Y$, Irisawa $Y$, Fujitani T. Ultrasound-guided erector spinae plane block elicts sensory loss around the lateral, but not the parasternal, portion of the thorax. J Clin Anesth 2018:47:84-85. [PubMed] DOI: 10.1016/j.jclinane.2018.03.023

7. Costache I, Pawa A, Abdallah FW. Paravertebral by proxy - time to redefine the paravertebral block. Anaesthesia 2018;73:1185-8. [PubMed] DOI: 10.1111/anae.14348 
8. Adhikary SD, Bernard S, Lopez H, Chin KJ. Erector spinae plane block versus retrolaminar block: a magnetic resonance imaging and anatomical study. Reg Anesth Pain Med 2018:43:756-62. [PubMed] DOI: 10.1097/AAP.0000000000000798

9. Ivanusic J, Konishi Y, Barrington MJ.. A cadaveric study investigating the mechanism of action of erector spinae blockade. Reg Anesth Pain Med 2018;43:567-71. [PubMed] DOI: 10.1097/AAP.0000000000000789

10. Fang B, Wang Z, Huang X. Ultrasound-guided preoperative single-dose erector spinae plane block provides comparable analgesia to thoracic paravertebral block following thoracotomy: a single center randomized controlled double-blind study. Ann Transl Med 2019;7: 174 . [PubMed] DOI: 10.21037/atm.2019.03.53

11. Zhang J, He Y, Wang S, Chen Z, Zhang Y, Gao Y, et al. The erector spinae plane block causes only cutaneous sensory loss on ipsilateral posterior thorax: a prospective observational volunteer study. BMC Anesthesiol 2020;20:88. [PubMed] DOI: 10.1186/s12871-020-01002-0

16. Elsharkawy $\mathrm{H}$, Maniker $\mathrm{R}$, Bolash $\mathrm{R}$, Kalasbail $\mathrm{P}$, Drake $\mathrm{RL}$, Elkassabany N. Rhomboid intercostal and subserratus plane block: a cadaveric and clinical evaluation. Reg Anesth Pain Med 2018;43:745-51. [PubMed] DOI: 10.1097/AAP.0000000000000824
12. Byrne $\mathrm{K}$, Smith $\mathrm{C}$. Human volunteer study examining the sensory changes of the thorax after an erector spinae plane block. Reg Anesth Pain Med 2020;45:7612. [PubMed] DOI: 10.1136/rapm-2019-101019

13. Lonnqvist PA, Karmakar MK, Richardson J, Moriggl B. Daring discourse: should the ESP block be renamed RIP II block? Reg Anesth Pain Med 2021;46:57-60. [PubMed] DOI: 10.1136/rapm-2020-101822

14. Costache I, de Neumann L, Ramnanan CJ, Goodwin SL, Pawa A, Abdallah FW, McCartney CJL. The midpoint transverse process to pleura (MTP) block: a new end-point for thoracic paravertebral block. Anaesthesia 2017;72:1230-6. [PubMed] DOI: 10.1111/anae.14004

15. Shibata Y, Kampitak W, Tansatit T. The novel costotransverse foramen block technique: distribution characteristics of injectate compared with erector spinae plane block. Pain Physician 2020;23(3):E305E314. [PubMed] 